\title{
Frequency and patterns of second-line resistance conferring mutations among MDR-TB isolates resistant to a second-line drug from eSwatini, Somalia and Uganda (2014-2016)
}

David Patrick Kateete ${ }^{1,2^{*}+}$ D, Rogers Kamulegeya ${ }^{1,2 \dagger}$, Edgar Kigozi ${ }^{1,2 \dagger}$, Fred Ashaba Katabazi ${ }^{1,2}$, Deus Lukoye ${ }^{3}$, Sindani Ireneaus Sebit ${ }^{4}$, Hergeye Abdi ${ }^{5}$, Peter Arube ${ }^{6}$, George William Kasule ${ }^{7}$, Kenneth Musisi ${ }^{7}$, Myalo Glen Dlamini ${ }^{8}$, Derrick Khumalo ${ }^{8}$ and Moses L. Joloba ${ }^{1,2^{*}}$

\begin{abstract}
Background: Pulmonary tuberculosis is a leading cause of morbidity and mortality in developing countries. Drug resistance, a huge problem in this contagious disease, is driven by point mutations in the Mycobacterium tuberculosis genome however, their frequencies vary geographically and this affects applicability of molecular diagnostics for rapid detection of resistance. Here, we report the frequency and patterns of mutations associated with resistance to second-line anti-TB drugs in multidrug-resistant (MDR) M. tuberculosis isolates from eSwatini, Somalia and Uganda that were resistant to a second-line anti-TB drug.

Methods: The quinolone resistance determining region (QRDR) of gyrA/gyrB genes and the drug resistance associated fragment of rrs gene from 80 isolates were sequenced and investigated for presence of drug resistance mutations. Of the 80 isolates, 40 were MDR, of which 28 (70\%) were resistant to a second-line anti-TB injectable drug, 18 (45\%) were levofloxacin resistant while 12 (30\%) were extensively drug resistant (XDR). The remaining 40 isolates were susceptible to anti-TB drugs. MIRU-VNTR analysis was performed for M/XDR isolates.

Results: We successfully sub-cultured 38 of the $40 \mathrm{M} / X D R$ isolates. The gyrA resistance mutations (Gly88Ala/Cys/Ala, Ala90Val, Ser91Pro, Asp94Gly/Asn) and gyrB resistance mutations (Asp500His, Asn538Asp) were detected in 72.2\% $(13 / 18)$ and 22.2\% (4/18) of the MDR and levofloxacin resistant isolates, respectively. Overall, drug resistance mutations in gyrA/gyrB QRDRs occurred in 77.8\% (14/18) of the MDR and levofloxacin resistant isolates. Furthermore, drug resistance mutations $11401 \mathrm{~g}$ and $\mathrm{g} 1484 \mathrm{t}$ in rrs occurred in 64.3\% (18/28) of the MDR isolates resistant to a second-line anti-TB injectable drug. Drug resistance mutations were not detected in drug susceptible isolates.

(Continued on next page)
\end{abstract}

\footnotetext{
* Correspondence: dkateete@chs.mak.ac.ug; moses.joloba@case.edu

${ }^{\dagger}$ David Patrick Kateete, Rogers Kamulegeya and Edgar Kigozi contributed equally to this work.

'Department of Immunology and Molecular Biology, School of Biomedical Sciences, Makerere University College of Health Sciences, Kampala, Uganda Full list of author information is available at the end of the article
}

(c) The Author(s). 2019 Open Access This article is distributed under the terms of the Creative Commons Attribution 4.0 International License (http://creativecommons.org/licenses/by/4.0/), which permits unrestricted use, distribution, and reproduction in any medium, provided you give appropriate credit to the original author(s) and the source, provide a link to the Creative Commons license, and indicate if changes were made. The Creative Commons Public Domain Dedication waiver (http://creativecommons.org/publicdomain/zero/1.0/) applies to the data made available in this article, unless otherwise stated. 


\begin{abstract}
(Continued from previous page)
Conclusions: The frequency of resistance mutations to second-line anti-TB drugs in MDR-TB isolates resistant to second line anti-TB drugs from eSwatini, Somalia and Uganda is high, implying that rapid molecular tests are useful in detecting second-line anti-TB drug resistance in those countries. Relatedly, the frequency of fluoroquinolone resistance mutations in gyrB/QRDR is high relative to global estimates, and they occurred independently of gyrA/QRDR mutations implying that their absence in panels of molecular tests for detecting fluoroquinolone resistance may yield false negative results in our setting.
\end{abstract}

Keywords: Tuberculosis, Fluoroquinolones, Aminoglycosides, gyrA gyrB, rrs, 165 rRNA, eSwatini, Somalia, Uganda

\section{Background}

Pulmonary tuberculosis (TB) remains a persistent global public health problem with a huge toll on populations in developing countries $[1,2]$. The drug-resistant forms of the disease especially multidrug resistant TB (MDR-TB) and extensively drug-resistant TB (XDR-TB) pose significant challenges for control of pulmonary TB and management of TB patients [3]. MDR-TB is caused by Mycobacterium tuberculosis strains that are resistant to the two powerful first-line anti-TB drugs, rifampicin and isoniazid. Extensively drug-resistant TB is "a rare type of MDR-TB that is resistant to a fluoroquinolone (FQ) and any of the three second-line injectable drugs: amikacin (AMK), kanamycin (KAN), and capreomycin (CAP)" [4]. MDR-TB and XDR-TB are very difficult to treat as the drug regimens are lengthy, toxic, and expensive $[5,6]$. TB control programs in many countries depend on early diagnosis and prompt identification of drug resistance $[3,7]$ and this is critical for reduction of resistance and preventing its spread in communities. Usually, the diagnosis of drug-resistant TB is achieved through sputum culturing accompanied by drug susceptibility testing (DST) of the bacilli, either in liquid or on solid culture media however, these conventional procedures are laborious, lengthy, expensive [7], and often not available in developing countries. As such, molecular susceptibility tests that can be performed within 48 hours are currently being deployed in African countries for rapid detection of resistance to anti-TB drugs, and the World Health Organization (WHO) has endorsed them for rapid detection of resistance to first-line and second-line anti-TB drugs [3]. Currently, the MTBDRsl test (Hain Lifescience, Nehren Germany) is the only available commercial test for rapid detection of resistance to second-line anti-TB drugs [8]; it targets resistance to FQs and second-line anti-TB injectable drugs AMK, KAN and CAP.

Spontaneous chromosomal point mutations are the main mechanism underlying drug resistance in TB [9] and a limited number of mutations accounts for majority of the phenotypic resistance to first- and second-line anti-TB drugs. Resistance to FQs, the most effective second-line anti-TB drugs used to treat MDR-TB, is associated with mutations in a short discrete region of the $\operatorname{gyr} A$ gene and less frequently gyr $B$, commonly referred to as the quinolone-resistance determining region (QRDR) [10]. Globally, up to $80 \%$ of FQ resistant $M$. tuberculosis is attributed to mutations in $g y r A / Q R D R$ mainly in codons 88, 90, 91 and 94 [10]. Furthermore, AMK, KAN and CAP resistance has been associated with mutations in the $16 S$ rRNA gene (rrs), between nucleotide positions 1400 and 1500 particularly mutations in positions 1401, 1402 and 1484; up to $87 \%$ of KAN and AMK resistance worldwide is attributed to these mutations (especially a1401g) [11, 12]. Additionally, KAN and CAP resistance can also be mediated by mutations in the eis and tlyA genes, respectively.It is now well-documented that the frequencies of drug resistance conferring mutations described above vary between $M$. tuberculosis geno-groups and geographically [13, 14], which pose a significant challenge in the development of sequence-based diagnostic tools and their applicability without a thorough evaluation of their performance with respect to setting. Case in point is the MTBDRsl test which, although it is generally useful in detecting resistance to second-line anti-TB drugs, it has varying sensitivity especially in detecting FQ resistance [15]. This creates need for characterizing $M$. tuberculosis isolates from different geographical regions to investigate frequencies of drug resistance conferring mutations. Here we describe these mutations in FQ (levofloxacin LXF, ofloxacin OFX, moxifloxacin MXF) resistant and AMK/KAN/CAP resistant $M$. tuberculosis isolates from eSwatini (formally Swaziland), Somalia and Uganda, all $\mathrm{TB}$ endemic countries with high prevalence of $\mathrm{HIV} / \mathrm{TB}$ co-infection $[16,21]$.

\section{Methods}

\section{Study setting and samples}

This was a cross-sectional study on stored isolates at Makerere University College of Health Sciences in Kampala, Uganda. Samples (sputum or isolates) were obtained from ongoing national surveillances for MDR$\mathrm{TB}$ and XDR-TB in the three countries of eSwatini, Somalia and Uganda. The isolates were cultured from 
patients with confirmed MDR-TB or presumptive XDR$\mathrm{TB}$ in which phenotypic or genotypic resistance to at least a second-line anti-TB drug was confirmed in the period between September 2014 and July 2016, Additional file 1: Table S1. A total of 40 MDR-TB and/or presumptive XDR-TB isolates met this criteria: 15 from eSwatini, 13 from Uganda and 12 from Somalia. This sample size reflects the WHO reports for proportions of MDR/RR-TB cases that are tested for resistance to second-line drugs in those countries [22], see references $[23,25]$ for details on country specific profiles for TB [23-25]. Furthermore, 40 isolates that were susceptible to first- and second-line anti-TB drugs were also included bringing the total number of isolates investigated to 80 (all non-repetitive isolates, one per patient). Samples/isolates from eSwatini and Somalia were shipped to Uganda and sub-cultured at the SupraNational Tuberculosis Reference Laboratory (SRL) in Kampala as described previously [16, 18, 21].

\section{Drug susceptibility testing}

Drug susceptibility testing (DST) was performed at the SRL in Kampala and repeated for second-line drug resistance at the Mycobacteriology (BSL-3) Laboratory, Makerere University College of Health Sciences. The isolates to be tested for resistance were sub-cultured for up to 14 days on Middlebrook 7H10 medium. Then, cultures were diluted to a McFarland standard of 0.5 by resuspending few healthy colonies in Middlebrook 7H9 medium with glycerol. We used the agar proportion method and antibiotic critical concentrations (CCs) recommended by the WHO for Middlebrook 7H10 medium i.e. LXF $1 \mu \mathrm{g} / \mathrm{ml}$, MXF $0.5 \mu \mathrm{g} / \mathrm{ml}$, AMK $2 \mu \mathrm{g} / \mathrm{ml}$, CAP $4 \mu \mathrm{g} / \mathrm{ml}$ and KAN 4 $\mu \mathrm{g} / \mathrm{ml}$ [26]. Middlebrook 7H10 agar quadrant plates were prepared by following standard microbiological procedures, and configured such that quadrant 1 was the drug free control while quadrants 2,3 and 4 contained antibiotics at the respective CCs indicated above. Plates were inoculated by following standard microbiological procedures i.e. with $100 \mu \mathrm{l}$ of $10^{-2}$ and $10^{-4}$ dilutions of inoculum adjusted to the turbidity equivalent to a McFarland standard of 0.5 , and the inoculum spread uniformly on the plate by tilting it (gently). The plates were sealed in plastic bags and incubated at $37^{\circ} \mathrm{C}$ for 21 days while being examined weekly. After 21-28 days of incubation, colonies on the plates were counted with the aid of a microscope. Antibiotic susceptibility was determined by comparing growth on drug containing quadrants to growth on drug free quadrant. An isolate was classified as resistant when colonies on the drug containing quadrant were more than $1 \%$ compared to the colonies on the drug free quadrant (control). Drug susceptible $M$. tuberculosis H37Rv strain and known M/XDR $M$. tuberculosis isolates were used for quality control.

\section{Genotyping}

Chromosomal DNA used as template in PCRs was extracted by following the $\mathrm{CTAB} /$ chloroform extraction method [27] with minor modifications as described previously [14]. Briefly, freshly cultured Mycobacterium cells were harvested and re-suspended in absolute ethanol (Sigma scientific, USA); the suspension was centrifuged at $16,000 \mathrm{~g}$ and the cell pellet re-suspended in $0.25 \mathrm{X}$ TrisEDTA (TE) buffer, which was used as template in PCRs. Prior to genotyping, isolates were confirmed to be $M$. tuberculosis by following an in-house PCR protocol described previously [28], in which they all tested positive for $M$. tuberculosis complex. To determine the genotypes, 15 loci Mycobacterial Interspersed Repetitive UnitsVariable Number of Tandem Repeats (MIRU-VNTR) genotyping was performed as described previously [29].

\section{PCRs and DNA sequencing}

The primers we used, and procedure we followed to amplify the targeted portions of genes associated with resistance to second-line anti-TB drugs i.e. $g y r A / g y r B$ QRDR and rrs were previously reported [7] (summarized in Additional file 1: Table S1, sheet 2). Primers were synthesized by Eurofins Genomics, Germany. PCRs were performed according to the protocol for the HotStar PCR kit (QIAGEN, Germany), in $60 \mu \mathrm{L}$ total volume as described previously [14]. Also, post-amplification analysis of the PCR products was as previously described [14]. Fifty microliters each of the PCR products was purified using the QIAmp DNA purification mini kit (QIAGEN) and sequenced by using the Sanger method at ACGT Inc. (Wheeling IL, USA).

\section{Sequence analysis and interpretation of mutations}

The amplicon-sequences were analysed first, by BLASTsearching at https://blast.ncbi.nlm.nih.gov/Blast.cgi to confirm they match the expected sequences in the $M$. tuberculosis genome. Furthermore, the gyrA/gyrB amplicon sequences were translated into amino acid sequences using MEGA6.06 software [30] or ExPASy online server http://web.expasy.org/translate/. Additionally, amplicon sequences were aligned to the $M$. tuberculosis $\mathrm{H} 37 \mathrm{Rv}$ RefSeq sequences (NCBI reference sequence NC_000962) for gyrA, gyrB, \& rrs promoter, using MEGA6.06 or BioEdit v7.2.5.0. To identify and interpret the mutations and determine whether they were conferring resistance to second-line anti-TB drugs, we used the ReSeqTB database http://www.reseqtb.org/Account/ Login?ReturnUrl=\%2F and current literature [7, 8, 31]. As well, for $\operatorname{gyr} B$, we used the "500-538" codon numbering system (1998) that most studies on FQ-resistance mutations have used, see Malik et al and Maruri et al, references [32, 33], respectively. The data was curated and presented as tables or percentages depending on 
frequencies of mutations that occurred in the examined sequences. $M$. tuberculosis strain $\mathrm{H} 37 \mathrm{Rv}$ is susceptible to anti-TB drugs and its $g y r A$, gyrB, and rrs sequences were regarded the "wild-type" during the analyses.

\section{Quality control}

Known FQ and KAN resistant MDR-TB isolates with well-characterized resistance conferring mutations were included as positive controls. Sequences were validated through nucleotide BLAST and translated BLAST searches to confirm that they match the expected genes in M. tuberculosis.

\section{Results}

\section{Second-line anti-TB drug resistance}

We successfully sub-cultured all the 40 drug susceptible isolates and 38 of the $40 \mathrm{MDR} /$ presumptive XDR-TB isolates that were resistant to a second-line anti-TB drug. All the isolates were investigated for mutations associated with resistance to second-line anti-TB drugs i.e. FQs (LXF, OFX, MXF), aminoglycosides (KAN, AMK) and CAP (a cyclic peptide). However for FQ resistance, only LXF and MXF resistance were considered in the analysis given the WHO's recommendation against testing for OFX resistance [26]. Generally KAN resistance was high among MDR isolates $(60.5 \%, 23 / 38)$ but comparable with respect to country of origin, Additional file 1: Table S1. AMK and CAP resistance was also high at $58 \%(22 / 38)$ and 50\% (19/38), respectively; as well, LXF/ MXF resistance was high $(47.4 \%, 18 / 38)$. Overall, $73.7 \%$ $(28 / 38)$ of the isolates were resistant to a second-line anti-TB injectable drug, of which 12 (31.6\%) were confirmed to be XDR as they were resistant to either LXF or MXF.

\section{M. tuberculosis genotypes}

Thirty four of the $38 \mathrm{M} / \mathrm{XDR}$ isolates were successfully genotyped by MIRU-VNTR analysis. The 13 isolates from Uganda were $M$. tuberculosis sub-lineages Uganda II (four isolates), Beijing (two isolates), and one isolate each for genotypes Uganda I, Delhi/CAS, NEW 1, LAM, West Africa 1 and S; one isolate from Uganda could not be genotyped. The 10 isolates from Somalia were $M$. tuberculosis Uganda I, West Africa II and Beijing (two isolates each) and one isolate each for genotypes Uganda II, West Africa I, and S; also, one isolate from Somalia could not be genotyped. Likewise, genotypes for the 15 isolates from eSwatini were diverse with Beijing (four isolates) and LAM (two isolates) being the most prevalent, and one isolate each for TUR, S, EAI, West Africa 1 , and NEW 1 -two isolates from eSwatini could not be genotyped, Additional file 1: Table S1. Overall, the most predominant genotypes in this study were $M$. tuberculosis Uganda (23.7\%, 9/38) and Beijing (21\%, 8/38),
Additional file 1: Table S1. Genotypes for the XDR isolates were Beijing (three), NEW-1, Uganda II, S, West Africa 2 (two isolates each) and Delhi/CAS (one isolate). Genotypes for the 40 drug-susceptible isolates were described elsewhere [34, 35].

\section{Drug resistance conferring mutations}

The most frequent drug resistance mutation in the MDR and LXF/MXF resistant isolates was Asp94Gly (33.3\%) and it was not detected in drug susceptible isolates, Table 1. Overall, drug resistance mutations in gyrA/ QRDR were detected in a total of 13 (72.2\%) MDR and LXF/MXF resistant isolates i.e. Asp94Gly (six isolates), Ala90Val (three isolates) as well as Gly88Cys, Gly88Ala, Ser91Pro and Asp94Asn, each occurring in one isolate, Table 1; Additional file 1: Table S1. For $\operatorname{gyrB} / \mathrm{QRDR}$, drug resistance mutations were detected in four $(22.2 \%)$ MDR and LXF/MXF resistant isolates i.e. Asp500His (3 isolates), Asn538Asp (1 isolate), Table 1 and Additional file 1: Table S1. Three of the four isolates (TC41789, TC84410 \& TC84702) with resistance mutations in $g y r B$ also possessed drug resistance mutations in $g y r A / Q R D R$ (Asp94Val / Ala90Val), Additional file 1: Table S1. Interestingly, isolate MG87552 from Somalia possessed only the mutation Asp500His and it lacked resistance mutations in gyrA/QRDR (Additional file 1: Table S1), implying that FQ resistance in this isolate was attributed to Asp500His. Additionally, all but one isolate with $\operatorname{gyr} B$ resistance mutations were from Uganda and all were $M$. tuberculosis Uganda genotype while the sole isolate from Somalia with a $\operatorname{gyrB}$ resistance mutation was $M$. tuberculosis West African 1. Overall, FQ resistance mutations either in $g y r A / Q R D R$ or $g y r B / Q R D R$ occurred in a total of $14(77.8 \%)$ MDR and LXF/MXF resistant isolates, Additional file 1: Table S1. Interestingly, 5 (27.8\%) MDR and LFX/MXF resistant isolates lacked known drug resistance mutations in $g y r A / g y r B$ QRDRs. Almost all the MDR and LXF/MXF resistant isolates had mutations outside the gyrA/QRDR but they also occurred in LXF/MXF susceptible isolates though at lower frequencies, Table 1.

By country, isolates with $\mathrm{FQ}$ resistance conferring mutations were distributed as follows: Uganda -six isolates with mutations $g y r A / A l a 90 \mathrm{Val}+$ gyrB/Asp500His (two isolates), gyrA/Asp94Gly + gyrB/Asn538Asp (one isolate), gyrA/Asp94Gly (two isolates) and gyrA/Ser91Pro (one isolate). Genotypes for these isolates were M. tuberculosis Uganda II (two isolates), Uganda I, West African 1, NEW-1 and Beijing (one isolate each). eSwatini -four isolates with mutations Asp94Gly, Asp94Asn, Asp94Gly, and Gly88Cys and their genotypes were Beijing (two isolates) and $\mathrm{S}$ (one isolate). Somalia -four isolates with gyrA mutations Gly88Ala, Asp94Gly, Ala90Val and gyrB mutation Asp500His; their genotypes were West African 
Table 1 Frequency and patterns of mutations associated with phenotypic resistance to second-line anti-TB drugs among MDR- and XDR-TB isolates resistant to a second-line TB drug from eSwatini, Somalia and Uganda (2014-2016)

\begin{tabular}{|c|c|c|c|c|c|c|c|c|c|c|}
\hline \multirow[t]{3}{*}{ Drug } & \multirow[t]{3}{*}{ Locus } & \multirow[t]{3}{*}{ Mutation } & \multicolumn{8}{|c|}{ Frequency (No. of isolates):- } \\
\hline & & & \multicolumn{4}{|c|}{ MDR \& LXF/MXF resistant $(n=18)$} & \multicolumn{4}{|c|}{$\begin{array}{l}\text { LXF/MXF susceptible } \\
\text { / Pan-susceptible }(n=40)\end{array}$} \\
\hline & & & $\begin{array}{l}\text { With } \\
\text { mutation }\end{array}$ & $\begin{array}{l}\text { Relative } \\
\text { Frequency } \\
\text { (\%) }\end{array}$ & $\begin{array}{l}\text { Without } \\
\text { mutation }\end{array}$ & $\begin{array}{l}\text { Relative Frequency } \\
(\%)\end{array}$ & $\begin{array}{l}\text { With } \\
\text { mutation }\end{array}$ & $\begin{array}{l}\text { Relative } \\
\text { Frequency } \\
(\%)\end{array}$ & $\begin{array}{l}\text { Without } \\
\text { mutation }\end{array}$ & $\begin{array}{l}\text { Relative } \\
\text { Frequency } \\
(\%) \\
\end{array}$ \\
\hline \multirow{29}{*}{$\begin{array}{l}\text { FLUOROQUINOLONES } \\
\text { (LXF, MXF) }\end{array}$} & \multirow{21}{*}{ gyrA } & Thr2Ala & 05 & 27.8 & 13 & 72.2 & 0 & 0 & 40 & 100 \\
\hline & & Thr2Arg & 05 & 27.8 & 13 & 72.2 & 0 & 0 & 40 & 100 \\
\hline & & Thr2Ala/Arg & 10 & 55.6 & 08 & 44.4 & 0 & 0 & 40 & 100 \\
\hline & & Asp3Glu & 04 & 22.2 & 14 & 77.8 & 02 & 5 & 38 & 95 \\
\hline & & Asp3Arg & 03 & 16.7 & 15 & 83.3 & 01 & 2.5 & 39 & 98 \\
\hline & & Asp3Val & 04 & 22.2 & 14 & 77.8 & 0 & 0 & 40 & 100 \\
\hline & & $\begin{array}{l}\text { Asp3Glu/Asp3Ar/ } \\
\text { Asp3Val }\end{array}$ & 11 & 61.1 & 07 & 40 & 0 & 0 & 40 & 100 \\
\hline & & Asp3Arg & 03 & 16.7 & 15 & 83.3 & 0 & 0 & 40 & 100 \\
\hline & & Asp3Val & 01 & 5.6 & 17 & 94.4 & 0 & 0 & 40 & 100 \\
\hline & & Gly88Cys & 01 & 5.6 & 17 & 94.4 & 0 & 0 & 40 & 100 \\
\hline & & Gly88Ala & 01 & 5.6 & 17 & 94.4 & 0 & 0 & 40 & 100 \\
\hline & & Gly88Ala/Cys & 02 & 11.1 & 16 & 89 & 0 & 0 & 40 & 100 \\
\hline & & Ala90Val & 03 & 16.7 & 15 & 83.3 & 0 & 0 & 40 & 100 \\
\hline & & Ser91Pro & 01 & 5.6 & 17 & 94.4 & 0 & 0 & 40 & 100 \\
\hline & & Asp94Gly & 06 & 33.3 & 12 & 66.7 & 0 & 0 & 40 & 100 \\
\hline & & Asp94Asn & 01 & 5.6 & 17 & 94.4 & 0 & 0 & 40 & 100 \\
\hline & & Asp94Asn/Gly & 07 & 39 & 11 & 61.1 & 0 & 0 & 40 & 100 \\
\hline & & $\begin{array}{l}\text { Gly88Ala/ } \\
\text { Gly88Cys/ } \\
\text { Gly88Ala/ } \\
\text { Ala90Val/ } \\
\text { Ser91Pro/ } \\
\text { Asp94Gly/ } \\
\text { Asp94Asn/ } \\
\text { Asp94Gly }\end{array}$ & 13 & 72.2 & 05 & 27.8 & 0 & 0 & 40 & 100 \\
\hline & & Arg128Ser & 01 & 5.6 & 17 & 94.4 & 01 & 2.5 & 39 & 98 \\
\hline & & Tyr129Asn & 04 & 22.2 & 14 & 77.8 & 01 & 2.5 & 39 & 98 \\
\hline & & Ala132Glu & 02 & 11.1 & 16 & 89 & 0 & 0 & 40 & 100 \\
\hline & \multirow[t]{8}{*}{ gyrB } & Gln608His & 01 & 5.6 & 17 & 94.4 & 0 & 0 & 40 & 100 \\
\hline & & Arg609Gly & 02 & 11.1 & 16 & 89 & 0 & 0 & 40 & 100 \\
\hline & & Tyr610Thr & 01 & 5.6 & 17 & 94.4 & 0 & 0 & 40 & 100 \\
\hline & & Arg485His & 01 & 5.6 & 17 & 94.4 & 0 & 0 & 40 & 100 \\
\hline & & Lys611Gln & 03 & 16.7 & 15 & 83.3 & 01 & 2.5 & 39 & 98 \\
\hline & & Asp500His & 03 & 16.7 & 15 & 83.3 & 0 & 0 & 40 & 100 \\
\hline & & Asn538Asp & 01 & 5.6 & 17 & 94.4 & 0 & 0 & 40 & 100 \\
\hline & & $\begin{array}{l}\text { Asp500His/ } \\
\text { Asn538Asp }\end{array}$ & 04 & 22.2 & 14 & 77.8 & 0 & 0 & 40 & 100 \\
\hline $\begin{array}{l}\text { KAN } \\
\text { CAP }\end{array}$ & \multirow[t]{4}{*}{ rss } & \multicolumn{5}{|c|}{ MDR \& KAN/CAP/AMK resistant $(n=28)$} & \multicolumn{4}{|c|}{$\begin{array}{l}\text { KAN/CAP/AMK susceptible / Pan-susceptible } \\
(n=40)\end{array}$} \\
\hline \multirow[t]{3}{*}{ AMK } & & a1401g & 17 & 60.7 & 11 & 39.3 & 0 & 0 & 40 & 100 \\
\hline & & $g 1484 t$ & 01 & 3.6 & 27 & 96.4 & 0 & 0 & 40 & 100 \\
\hline & & a1401g/g1484 & 18 & 64.3 & 10 & 35.7 & 0 & 0 & 40 & 100 \\
\hline
\end{tabular}


Table 1 Frequency and patterns of mutations associated with phenotypic resistance to second-line anti-TB drugs among MDR- and XDR-TB isolates resistant to a second-line TB drug from eSwatini, Somalia and Uganda (2014-2016) (Continued)

\begin{tabular}{|c|c|c|c|c|c|c|c|c|c|c|}
\hline \multirow[t]{3}{*}{ Drug } & \multirow[t]{3}{*}{ Locus } & \multirow[t]{3}{*}{ Mutation } & \multicolumn{8}{|c|}{ Frequency (No. of isolates):- } \\
\hline & & & \multicolumn{4}{|c|}{ MDR \& LXF/MXF resistant $(n=18)$} & \multicolumn{4}{|c|}{$\begin{array}{l}\text { LXF/MXF susceptible } \\
\text { / Pan-susceptible }(n=40)\end{array}$} \\
\hline & & & $\begin{array}{l}\text { With } \\
\text { mutation }\end{array}$ & $\begin{array}{l}\text { Relative } \\
\text { Frequency } \\
\text { (\%) }\end{array}$ & $\begin{array}{l}\text { Without } \\
\text { mutation }\end{array}$ & $\begin{array}{l}\text { Relative Frequency } \\
\text { (\%) }\end{array}$ & $\begin{array}{l}\text { With } \\
\text { mutation }\end{array}$ & $\begin{array}{l}\text { Relative } \\
\text { Frequency } \\
\text { (\%) }\end{array}$ & $\begin{array}{l}\text { Without } \\
\text { mutation }\end{array}$ & $\begin{array}{l}\text { Relative } \\
\text { Frequency } \\
\text { (\%) }\end{array}$ \\
\hline & & \multicolumn{9}{|l|}{$\mathbf{t}^{\mathrm{a}}$} \\
\hline & & $\mathrm{g} 1158 \mathrm{t}$ & 01 & 3.6 & 27 & 96.4 & 0 & 0 & 40 & 100 \\
\hline
\end{tabular}

High confidence mutations are presented in boldface font

${ }^{a}$ Number of isolates with any of these high confidence mutations

2 (two isolates), S (one isolate) and Uganda I (the isolate with $g y r B /$ Asp500His).

Furthermore, almost all the $28 \mathrm{MDR}-\mathrm{TB}$ isolates resistant to a second-line anti-TB injectable drug (KAN, CAP, AMK) had mutations in the rrs gene $(96.4 \%, 27 / 28)$ with a1401g being the most frequent $(60.7 \%, 17 / 28)$ while g1484 $t$ occurred in only one isolate, Table 1 . Altogether, the drug resistance mutations $a 1401 g$ and $g 1484 t$ combined occurred in 18 (64.3\%) MDR and KAN/CAP/ AMK resistant isolates while 10 (35.7\%) isolates lacked known drug resistance conferring mutations in rss. Similar to $g y r A$, the rrs mutations were more frequent in the Beijing genotype and by country, they were proportionally more frequent in isolates from eSwatini and Uganda. None of the 40 drug susceptible isolates harboured resistance conferring mutations to second-line anti-TB drugs. Of note, several lineage-specific polymorphisms and mutations that are not known to confer resistance to second-line drugs were detected but these are not discussed (Additional file 1: see Table S1).

\section{Discussion}

In this study, the combined frequency of known drug resistance conferring mutations in MDR-TB isolates that were resistant to a second-line anti-TB drug from eSwatini, Somalia and Uganda was moderately high i.e. $64.3 \%$ for injectable drugs and $77.8 \%$ for FQs, and comparable to reported frequencies for these mutations worldwide [7, 31]. However, our rates are slightly lower compared to some reports [7, 8, 31, 36] but such discrepancies are commonly reported even in studies from the same region or country $[37,38]$. Several investigators worldwide also reported low individual frequencies for mutations especially gyrA/Asp94Asn and gyrA/Asp94Gly in FQ resistant $M$. tuberculosis isolates e.g. 25\% in Shanghai China [39] and 10\% (2/20) in a recent study in Uganda [40]. In one systematic review of the gyrase mutations associated with FQ resistance in $M$. tuberculosis, only 780 (64\%) of the 1220 FQ resistant isolates had mutations, of which those affecting the drug resistance gyrA codons 90 , 91 or 94 were present in only $54 \%(654 / 1220)$ of the resistant isolates [33]. In India, only $17 \%$ (25/146) of the MDR isolates had mutations in gyrA/QRDR with the commonest being Asp94Gly (48.1\%, 13/27) [41]. Generally, the observed low frequencies for individual resistance conferring mutations relative to global estimates could be attributed to mutations or resistance mechanisms in other key genes we did not investigate e.g. eis for second-line anti-TB injectable drugs [31].

In this study, the overall frequency $(77.8 \%)$ of drug resistance conferring mutations in $g y r A$ and gyrB of FQ resistant MDR-TB isolates was high and almost similar to global estimates for frequencies of these mutations in FQ resistant M. tuberculosis isolates i.e. 80\%-92\% [42, 43]. Interestingly, the frequency (22.2\%) of resistance mutations Asp500His and Asn538Asp in $g y r B / Q R D R$ in this study was higher than global estimates for these mutations i.e. $\leq 2 \%$ [42] or $\leq 5 \%$ [43]. Generally, gyrB/QRDR resistance mutations especially Asn538Asp, Asp500His and Arg485His were confirmed to confer cross-resistance to FQs in $M$. tuberculosis [32] but they are considered to be rare and occur at much lower frequencies (i.e. $1 \%-2 \%$ in FQ resistant $M$. tuberculosis isolates [42]). Whether gyrB resistance mutations are more frequent in African populations as suggested by this study requires further investigation.

Usually, drug resistance mutations in $g y r B / Q R D R$ occur in association with $g y r A / Q R D R$ resistance mutations and they mostly affect codons 500 and 538 [42, 43]. The association of $g y r B$ resistance mutations with $g y r A$ mutations has made it difficult to evaluate the contribution of $g y r B$ mutations to FQ resistance [42]. Interestingly, in this study the gyrB mutation Asp500His occurred in a FQ resistant isolate independently of resistance mutations in $g y r A$ / QRDR, implying that gyrB mutations in our setting individually confer resistance to FQs, as experimentally validated by Malik et al [32]. Relatedly, in a systematic review of the frequencies and geographic distribution of $g y r A$ and gyrB mutations associated with FQ resistance in clinical M. tuberculosis isolates [42], it was reported that $g y r B$ mutations indeed occurred independently of gyrA mutations in certain settings perhaps explaining phenotypic resistance in isolates lacking mutations in gyrA/QRDR [42]. 
Furthermore, we also detected mutations in $g y r A / g y r B$ genes that are not drug resistance conferring e.g. Thr2Ala/Arg, Asp3Val, Thr4Gly, Ala132Asp, Ala132Glu and Arg133Gln, and they were not included in systematic surveys of confidence-graded mutations associated with phenotypic resistance to FQs [31]. As well, neutral polymorphisms in $g y r A$, which are not drug resistance conferring e.g. Glu21Gln, Thr80Ala, Ser95Thr, etc. are frequently observed in both FQ resistant and FQ susceptible isolates $[44,45]$. The Thr80Ala polymorphism has been detected in African isolates [46] and it is regarded a lineage-specific marker for $M$. tuberculosis sub-lineage Uganda strains [44] that are prevalent in Uganda and in countries in central Africa [47]. In the current study, this polymorphism occurred in only " $M$. tuberculosis Uganda" isolates but further studies are required to demonstrate its usefulness as a marker for this genotype. Although neutral polymorphisms in resistance associated genes of $M$. tuberculosis are generally not resistance conferring [44], further research is required to ascertain they have no role in resistance. For example, the simultaneous occurrence of Thr80Ala and Ala90Gly polymorphisms in gyrA/QRDR led to a FQ hyper-susceptibility phenotype in laboratory-based experiments [32, 48, 49]. With the increase in sequencing data for M. tuberculosis isolates from across the globe, it would be interesting to investigate the frequency of isolates with dual mutations Thr80Ala \& Ala90Gly in clinically circulating TB strains and ascertain the association between carriage of the dual mutations Thr80Ala \& Ala90Gly with FQ hypersusceptibility. These dual mutations occurred in two isolates in this study (TC84410 \& TC84702) and they also occurred in nine clinical $M$. tuberculosis isolates in Congo-Brazzaville [50], implying they could be prevalent in Africa.

\section{Limitations}

This study had certain limitations: First, we characterized MDR isolates that were resistant to second line anti-TB drugs from eSwatini, Somalia and Uganda however, the total number of isolates that met this criteria $(n=40)$ was small relative to the TB burden in those countries. As such, the true frequencies of drug resistance conferring mutations to second-line anti-TB drugs could be higher than we have reported if a larger sample size is used. However, the fact that the reported isolates were collected over a long period of time, studies with larger sample sizes require a lot of time. Second, our findings may not be generalizable especially for Somalia and Uganda as only a small fraction of M/XDR cases (10\%-20\%) are tested for resistance to second-line anti-TB drugs [23, 25]. Nevertheless, our findings provide key insight into the performance of molecular DST methods in detecting resistance to second-line anti-TB drugs in eSwatini, Somalia and Uganda.

\section{Conclusions}

The frequencies of drug resistance conferring mutations to second-line anti-TB drugs in MDR isolates that are resistant to second line anti-TB drugs from eSwatini, Somalia and Uganda is high especially to FQs, implying that rapid molecular tests e.g. MTBDRplus/sl line probe assay (LPA) that has great promise in detecting MDR isolates [8], as well as DNA sequencing, are useful in detecting FQ and AMK/KAN/CAP resistant $M$. tuberculosis in those countries. Relatedly, the frequency of FQ resistance conferring mutations in $\operatorname{gyr} B$ was high relative to global estimates, and occurred independently of $g y r A$ mutations implying that absence of $g y r B / Q R D R$ resistance mutations in panels for rapid molecular tests for detection of FQ resistance could lead to false negative results in this setting.

\section{Additional file}

Additional file 1: Table S1. Sheet 1 -mutations in drug resistance associated genes/loci among MDR M. tuberculosis isolates from Uganda, Somalia and eSwatini that were resistant to any of: Ofloxacin,

levofloxacin, moxifloxacin, kanamycin, capreomycin, amikacin; Sheet 2 primers and sequences used. (XLSX $20 \mathrm{~kb}$ )

\section{Abbreviations}

AMK: Amikacin; BSL-3: Biosafety Level 3; CAP: Capreomycin; DST: Drug susceptibility testing; FQs: Fluoroquinolones; KAN: Kanamycin; LPA: Line probe assay; LXF: Levofloxacin; MDR-TB: Multidrug resistant tuberculosis; MEGA6.06: Molecular Evolutionary Genetics Analysis Version 6.0; MIRUVNTR: Mycobacterial Interspersed Repetitive Units-Variable Number of Tandem Repeats analysis; MXF: Moxifloxacin; NCBI: National Center for Biotechnological Information; OFX: Ofloxacin; QRDR: Quinolone-resistance determining region; rrs: 165 ribosomal RNA gene; RR-TB: Rifampicin resistant TB; TE: Tris-EDTA buffer solution, pH 8.0; XDR-TB: Extensively drug-resistant tuberculosis

\section{Acknowledgements \\ We thank staff at the National Tuberculosis Reference Laboratory/SRL Uganda for technical assistance. We also thank Joshua Mandre \& Allen Mukhwana of MUII-Plus/UVRI, and Carolyne Atugonza, Moses Okee \& Geraldine Nalwadda of Makerere University College of Health Sciences for administrative support.}

\section{Authors' contributions \\ DPK conceived and designed the study. KR, EK and FAK sub-cultured the isolates, performed the molecular experiments, analyzed and interpreted the data (under supervision of DPK \& ML), and wrote the first draft of the manuscript. SIS, HA, PA, ML, KGW, KM, DL, DGM, and DK cultured/sub-cultured the sputum samples/isolates, performed and interpreted phenotypic and genotypic (LPA) drug susceptibility testing. All authors read and approved the final manuscript.}

\section{Funding}

This work was supported through a DELTAS Africa Initiative [grant\# 107743/ Z/15/Z]. The DELTAS Africa Initiative is an independent funding scheme of the African Academy of Sciences (AAS), Alliance for Accelerating Excellence in Science in Africa (AESA), and supported by the New Partnership for Africa's Development Planning and Coordinating Agency (NEPAD Agency) with funding from the Wellcome Trust (Grant no. 107743) and the UK Government. The funders had no role in study design, data collection and analysis, decision to publish, or preparation of the manuscript. DPK was a 
recipient of a Postdoctoral Fellowship award from the Makerere University Infection and Immunity Programme (MUII-Plus) at MRC/UVRI, Entebbe.

\section{Availability of data and materials}

The datasets used and/or analysed during the current study are available from the corresponding authors on reasonable request.

\section{Ethics approval and consent to participate}

This study was approved by the Higher Degrees Research and Ethics Committee of the School of Biomedical Sciences, Makerere University College of Health Sciences. This committee permitted the use of archived $M$. tuberculosis isolates from patients who participated in the Drug Resistance Surveys that investigated the phenotypic levels and patterns of resistance to first- and second-line anti-TB drugs among new and previously treated sputum smear-positive pulmonary TB patients in Uganda, Somalia and eSwatini. The national/sub-national drug resistance surveys obtained written informed consent (or assent, where applicable) from all the participants for sample storage and use of stored samples in further studies.

\section{Consent for publication}

Not applicable

\section{Competing interests}

The authors declare that they have no competing interests

\section{Author details}

'Department of Immunology and Molecular Biology, School of Biomedical Sciences, Makerere University College of Health Sciences, Kampala, Uganda. ${ }^{2}$ Department of Medical Microbiology, School of Biomedical Sciences, Makerere University College of Health Sciences, Kampala, Uganda. ${ }^{3}$ National Tuberculosis/Leprosy Program, Ministry of Health Uganda, Kampala, Uganda. ${ }^{4} \mathrm{WHO}$, Hargeisa, Somaliland, Somalia. ${ }^{5}$ Ministry of Health, Hargeisa, Somaliland, Somalia. ${ }^{6}$ World Vision International, Nairobi, Kenya. ${ }^{7}$ National Tuberculosis Reference Laboratory, Kampala, Uganda. ${ }^{8}$ National TB Reference Laboratory / eSwatini Health Laboratory Services, Ministry of Health, Hospital Hill Mbabane, Mbabane, eSwatini.

\section{Received: 14 February 2019 Accepted: 3 July 2019}

Published online: 10 July 2019

\section{References}

1. World Health Organization (WHO): The top 10 causes of death. $2017 \mathrm{http}: / /$ www.who.int/mediacentre/factsheets/fs310/en/. 29 May 2017.

2. World Health Organization (WHO): Leading causes of death by economy income group. 2017 https://www.who.int/news-room/fact-sheets/detail/the-top-10causes-of-death.

3. World Health Organization (WHO): Tuberculosis. 2017 http://www.who.int/ mediacentre/factsheets/fs104/en/. 29 May 2017.

4. Centers for Disease Control and Prevention (CDC). Emergence of Mycobacterium tuberculosis with extensive resistance to second-line drugs-worldwide, 2000-2004. MMWR Morb Mortal Wkly Rep. 2006;55(11):301-5.

5. Manjelievskaia J, Erck D, Piracha S, Schrager L. Drug-resistant TB: deadly, costly and in need of a vaccine. Trans R Soc Trop Med Hyg. 2016;110(3): 186-91.

6. Seung KJ, Keshavjee S, Rich ML. Multidrug-Resistant Tuberculosis and Extensively Drug-Resistant Tuberculosis. Cold Spring Harb Perspect Med. 2015;5(9):a017863.

7. Campbell PJ, Morlock GP, Sikes RD, Dalton TL, Metchock B, Starks AM, Hooks DP, Cowan LS, Plikaytis BB, Posey JE. Molecular detection of mutations associated with first- and second-line drug resistance compared with conventional drug susceptibility testing of mycobacterium tuberculosis. Antimicrob Agents Chemother. 2011;55(5):2032-41.

8. Tagliani E, Cabibbe AM, Miotto P, Borroni E, Toro JC, Mansjo M, Hoffner S, Hillemann D, Zalutskaya A, Skrahina A, et al. Diagnostic performance of the new version (v2.0) of GenoType MTBDRsl assay for detection of resistance to fluoroquinolones and second-line injectable drugs: a multicenter Study. J Clin Microbiol. 2015;53(9):2961-9.

9. Zhang Y, Yew W. Mechanisms of drug resistance in Mycobacterium tuberculosis [State of the art series. Drug-resistant tuberculosis. Edited by CY. Chiang. Number 1 in the series]. Int J Tuberc Lung Dis. 2009;13(11): $1320-30$.
10. Zhao X, Xu C, Domagala J, Drlica K. DNA topoisomerase targets of the fluoroquinolones: a strategy for avoiding bacterial resistance. Proc Natl Acad Sci. 1997;94(25):13991-6.

11. Wongsrichanalai C, Varma JK, Juliano JJ, Kimerling ME, MacArthur JR. Extensive drug resistance in malaria and tuberculosis. Emerg Infect Dis. 2010;16(7):1063.

12. Almeida Da Silva PE, Palomino JC. Molecular basis and mechanisms of drug resistance in Mycobacterium tuberculosis: classical and new drugs. J Antimicrob Chemother. 2011;66(7):1417-30.

13. Mustafa S, Javed H, Hashmi J, Jamil N, Tahir Z, Akhtar AM. Emergence of mixed infection of Beijing/Non-Beijing strains among multi-drug resistant Mycobacterium tuberculosis in Pakistan. 3 Biotech. 2016;6(1):108.

14. Kigozi E, Kasule GW, Musisi K, Lukoye D, Kyobe S, Katabazi FA, Wampande EM, Joloba ML, Kateete DP. Prevalence and patterns of rifampicin and isoniazid resistance conferring mutations in Mycobacterium tuberculosis isolates from Uganda. PLOS ONE. 2018;13(5):e0198091.

15. Theron G, Peter J, Richardson M, Warren R, Dheda K, Steingart KR. GenoType ${ }^{\circledast}$ MTBDRsl assay for resistance to second-line anti-tuberculosis drugs. Cochrane Database Syst Rev. 2016;(9):1-4.

16. Lukoye D, Adatu F, Musisi K, Kasule GW, Were W, Odeke R, Kalamya JN, Awor A, Date A, Joloba ML. Anti-Tuberculosis Drug Resistance among New and Previously Treated Sputum Smear-Positive Tuberculosis Patients in Uganda: Results of the First National Survey. PLoS One. 2013;8(8):e70763.

17. Lukoye D, Cobelens FGJ, Ezati N, Kirimunda S, Adatu FE, Lule JK, Nuwaha F, Joloba ML. Rates of Anti-Tuberculosis Drug Resistance in Kampala-Uganda Are Low and Not Associated with HIV Infection. PLoS One. 2011;6(1):e16130.

18. Lukoye D, Ssengooba W, Musisi K, Kasule GW, Cobelens FG, Joloba M, Gomez GB. Variation and risk factors of drug resistant tuberculosis in sub-Saharan Africa: a systematic review and meta-analysis. BMC Public Health. 2015;15:291.

19. Musa BM, Adamu AL, Galadanci NA, Zubayr B, Odoh CN, Aliyu MH. Trends in prevalence of multi drug resistant tuberculosis in sub-Saharan Africa: A systematic review and meta-analysis. PLoS One. 2017;12(9):e0185105.

20. Sanchez-Padilla E, Dlamini T, Ascorra A, Rüsch-Gerdes S, Tefera ZD, Calain P, de la Tour R, Jochims F, Richter E, Bonnet M. High Prevalence of Multidrug-Resistant Tuberculosis, Swaziland, 2009-2010. Emerg Infect Dis. 2012;18(1):29-37.

21. Sindani I, Fitzpatrick C, Falzon D, Suleiman B, Arube P, Adam I, Baghdadi S, Bassili A, Zignol M. Multidrug-Resistant Tuberculosis, Somalia, 2010-2011. Emerg Infect Dis. 2013;19(3):478-80.

22. World Health Organization (WHO): Global tuberculosis report 2016. 2016.

23. World Health Organization (WHO): Tuberculosis country profile -Uganda. 2017 https:/extranet.who.int/sree/Reports?op=Replet\&name=NHO_HQ Reports/G2/PROD/EXT/TBCountryProfile\&ISO2=UG\&outtype=PDF .

24. World Health Organization (WHO): Tuberculosis country profile -Eswatini. 2017 https://extranet.who.int/sree/Reports?op=Replet\&name=NHO_HQ_ Reports/G2/PROD/EXT/TBCountryProfile\&ISO2=SZ\&outtype=pdf.

25. World Health Organization (WHO): Tuberculosis country profile -Somalia. 2017 https://extranet.who.int/sree/Reports?op=Replet\&name=NHO_HQ_ Reports/G2/PROD/EXT/TBCountryProfile\&ISO2=so\&outtype=pdf.

26. World Health Organization (WHO): Technical Report on critical concentrations for drug susceptibility testing of medicines used in the treatment of drug-resistant tuberculosis. 2018.

27. van Helden PD, Victor TC, Warren RM, van Helden EG. Isolation of DNA from Mycobacterium tubercolosis. In: Parish T, Stoker NG, editors. Mycobacterium tuberculosis Protocols. Totowa: Humana Press; 2001. p. 19-30.

28. Muhumuza J, Asiimwe BB, Kayes S, Mugyenyi P, Whalen C, Mugerwa RD, Boom H, Eisenach KD, Joloba ML. Introduction of an in-house PCR for routine identification of $\mathrm{M}$. tuberculosis in a low-income country. Int J Tuberc Lung Dis. 2006;10(11):1262-7.

29. Nabyonga L, Kateete DP, Katabazi FA, Odong PR, Whalen CC, Dickman KR, Joloba ML. Determination of circulating Mycobacterium tuberculosis strains and transmission patterns among pulmonary TB patients in Kawempe municipality, Uganda, using MIRU-VNTR. BMC Res Notes. 2011;4:280.

30. Tamura K, Stecher G, Peterson D, Filipski A, Kumar S. MEGA6: molecular evolutionary genetics analysis version 6.0. Mol Biol Evol. 2013;30(12):2725-9.

31. Miotto P, Tessema B, Tagliani E, Chindelevitch L, Starks Angela M, Emerson C, Hanna D, Kim PS, Liwski R, Zignol M, et al. A standardised method for interpreting the association between mutations and phenotypic drug resistance in Mycobacterium tuberculosis. Eur Respir J. 2017;50(6):1701354.

32. Malik S, Willby M, Sikes D, Tsodikov OV, Posey JE. New Insights into Fluoroquinolone Resistance in Mycobacterium tuberculosis: Functional Genetic Analysis of gyrA and gyrB Mutations. PLoS One. 2012;7(6):e39754. 
33. Maruri F, Sterling TR, Kaiga AW, Blackman A, van der Heijden YF, Mayer C, Cambau E, Aubry A. A systematic review of gyrase mutations associated with fluoroquinolone-resistant Mycobacterium tuberculosis and a proposed gyrase numbering system. J Antimicrob Chemother. 2012;67(4):819-31.

34. Ezati N, Lukoye D, Wampande EM, Musisi K, Kasule GW, Cobelens FGJ, Kateete DP, Joloba ML. The Mycobacterium tuberculosisUganda II family and resistance to first-line anti-tuberculosis drugs in Uganda. BMC Infect Dis. 2014;14(1):703.

35. Lukoye D, Katabazi FA, Musisi K, Kateete DP, Asiimwe BB, Okee M, Joloba ML, Cobelens FGJ. The T2 Mycobacterium tuberculosis Genotype, Predominant in Kampala, Uganda, Shows Negative Correlation with Antituberculosis Drug Resistance. Antimicrob Agents Chemother. 2014;58(7):3853-9.

36. Zhang Z, Lu J, Wang Y, Pang Y, Zhao Y. Prevalence and molecular characterization of fluoroquinolone-resistant Mycobacterium tuberculosis isolates in China. Antimicrob Agents Chemother. 2014:58(1):364-9.

37. Ginsburg AS, Grosset JH, Bishai WR. Fluoroquinolones, tuberculosis, and resistance. Lancet Infect Dis. 2003;3(7):432-42.

38. Lee AS, Tang LL, Lim IH, Wong SY. Characterization of pyrazinamide and ofloxacin resistance among drug-resistant Mycobacterium tuberculosis isolates from Singapore. Int J Infect Dis. 2002;6(1):48-51.

39. Zhu C, Zhang Y, Shen Y, Siu GKH, Wu W, Qian X, Deng G, Xu Y, Lau R, Fan $X$. Molecular characterization of fluoroquinolone-resistant Mycobacterium tuberculosis clinical isolates from Shanghai, China. Diagn Microbiol Infect Dis. 2012;73(3):260-3.

40. Rosales-Klintz S, Jureen P, Zalutskayae A, Skrahina A, Xu B, Hu Y, PinedaGarcia L, Merza MA, Muntean I, Bwanga F, et al. Drug resistance-related mutations in multidrug-resistant Mycobacterium tuberculosis isolates from diverse geographical regions. Int J Mycobacteriol. 2012;1(3):124-30.

41. Singhal R, Reynolds PR, Marola JL, Epperson LE, Arora J, Sarin R, Myneedu VP, Strong M, Salfinger M. Sequence Analysis of Fluoroquinolone ResistanceAssociated Genes gyrA and gyrB in Clinical Mycobacterium tuberculosis Isolates from Patients Suspected of Having Multidrug-Resistant Tuberculosis in New Delhi, India. J Clin Microbiol. 2016;54(9):2298-305.

42. Avalos E, Catanzaro D, Catanzaro A, Ganiats T, Brodine S, Alcaraz J, Rodwell T. Frequency and Geographic Distribution of gyrA and gyrB Mutations Associated with Fluoroquinolone Resistance in Clinical Mycobacterium Tuberculosis Isolates: A Systematic Review. PLoS One. 2015:10(3):e0120470.

43. Nosova EY, Bukatina AA, Isaeva YD, Makarova MV, Galkina KY, Moroz AM. Analysis of mutations in the gyrA and gyrB genes and their association with the resistance of Mycobacterium tuberculosis to levofloxacin, moxifloxacin and gatifloxacin. J Med Microbiol. 2013;62(Pt 1):108-13.

44. Feuerriegel S, Koser CU, Niemann S. Phylogenetic polymorphisms in antibiotic resistance genes of the Mycobacterium tuberculosis complex. J Antimicrob Chemother. 2014;69(5):1205-10.

45. Kim H, Nakajima C, Kim YU, Yokoyama K, Suzuki Y. Influence of lineagespecific amino acid dimorphisms in GyrA on Mycobacterium tuberculosis resistance to fluoroquinolones. Jpn J Infect Dis. 2012;65(1):72-4.

46. Umubyeyi AN, Rigouts L, Shamputa IC, Fissette K, Elkrim Y, De Rijk P, Struelens M, Portaels F. Limited fluoroquinolone resistance among Mycobacterium tuberculosis isolates from Rwanda: results of a national survey. J Antimicrob Chemother. 2007;59(5):1031-3.

47. Stucki D, Brites D, Jeljeli L, Coscolla M, Liu Q, Trauner A, Fenner L, Rutaihwa L, Borrell S, Luo T, et al. Mycobacterium tuberculosis lineage 4 comprises globally distributed and geographically restricted sublineages. Nat Genet. 2016;48(12): 1535-43.

48. Aubry A, Veziris N, Cambau E, Truffot-Pernot C, Jarlier V, Fisher LM. Novel gyrase mutations in quinolone-resistant and -hypersusceptible clinical isolates of Mycobacterium tuberculosis: functional analysis of mutant enzymes. Antimicrob Agents Chemother. 2006;50(1):104-12.

49. Palomino JC, Martin A. Drug Resistance Mechanisms in Mycobacterium tuberculosis. Antibiotics (Basel, Switzerland). 2014:3(3):317-40.

50. Aubry A, Sougakoff W, Bodzongo P, Delcroix G, Armand S, Millot G, Jarlier V, Courcol R, Lemaitre N. First evaluation of drug-resistant Mycobacterium tuberculosis clinical isolates from Congo revealed misdetection of fluoroquinolone resistance by line probe assay due to a double substitution T80A-A90G in GyrA. PLoS One. 2014;9(4):e95083.

\section{Publisher's Note}

Springer Nature remains neutral with regard to jurisdictional claims in published maps and institutional affiliations.

\section{Ready to submit your research? Choose BMC and benefit from}

- fast, convenient online submission

- thorough peer review by experienced researchers in your field

- rapid publication on acceptance

- support for research data, including large and complex data types

- gold Open Access which fosters wider collaboration and increased citations

- maximum visibility for your research: over $100 \mathrm{M}$ website views per year

At $\mathrm{BMC}$, research is always in progress.

Learn more biomedcentral.com/submissions 